\title{
Pensando emoções a partir de uma perspectiva interseccional: as geografias emocionais das campanhas eleitorais equatorianas*
}

\author{
Thinking Emotions from an Intersectional Perspective: The Emotional \\ Geographies of Ecuadorian Electoral Campaigns
}

\author{
Carolin Schurr \\ Universidade de Berna - Suíça \\ schurr@giub.unibe.ch
}

\section{Resumo}

Observando a campanha eleitoral no Equador em 2009, este artigo defende que as emoções são um ingrediente fundamental em qualquer tipo de campanha política. É difícil imaginar comícios públicos, sem gritos, multidão cantando e batendo palmas, os que expressam entusiasmo, simpatia, ou raiva. Sugiro que essas emoções resultam de, e ao mesmo tempo respondem, a desigualdades generificadas, racializadas e agrupadas dentro da sociedade pós-colonial do Equador. Sentimentos de injustiça, raiva, vergonha, orgulho e, assim por diante estão enraizados na hierarquia de identidades em uma ordem social (pós) colonial. Apesar de tradicionalmente homens branco mestiços estarem dominado o cenário político, desde a década de 1990, mulheres, populações indígenas e afros equatorianas ganharam visibilidade política através da introdução de uma lei de cotas de gênero e da fundação de partidos indígenas. Este trabalho pretende analisar as emoções desses 'novos' indivíduos políticos colocados em exposição, com foco em como suas performances emocionais respondem e desafiam os sistemas interseccionais de opressão, tais como racismo, classismo e heteronormativismo. Ao fazer isso, o trabalho contribui para uma alternativa, o engajamento não universalizado com as geografias da emoção que incorporam as noções de raça, poder e gênero.

Palavras-chave: geografias emocionais; política; interseccionalidade; pós-colonialismo; identidade.

\begin{abstract}
Looking at Ecuador's electoral campaign in 2009, this paper argues that emotions are a key ingredient in any kind of political campaigning. It is hard to imagine public rallies without the shouting, crowd chanting, and clapping that express enthusiasm, sympathy or anger. I suggest that these emotions result from and at the same time respond to gendered, racialised and classed inequalities within Ecuador's postcolonial society. Feelings of injustice, anger, shame, pride and so on are rooted in the hierarchy of identities in a (post)colonial societal order. While traditionally white-mestizo men have dominated the political stage, since the 1990s women, indigenous and Afro-Ecuadorian people have gained political visibility through the introduction of a gender quota law and the foundation of indigenous parties. This paper sets out to examine the emotions these 'new' political subjects put on display, focusing on how their emotional performances respond to and challenge intersectional systems of oppression such as racism, classism and heteronormativism. In so doing, the paper contributes to an alternative, nonuniversalising engagement with the geographies of emotion that incorporates notions of power, race, and gender.
\end{abstract}

Keywords: emotional geographies; politics; intersectionality; postcoloniality; identity. 


\section{Geografias emocionais dos espaços políticos locais do Equador}

\begin{abstract}
O ponto é que a democracia sem discussão impetuosa, sem floreios retóricos, e sem o compartilhamento de risos, raiva, ou dor é democracia sem vitalidade (HOGGETT, 2009).
\end{abstract}

Estudiosos, de diferentes áreas, estão cada vez mais conscientes de que as emoções importam na constituição do político. Em 'Zorn und Zeit', o filósofo alemão Peter Sloterdijk (2008) examina como a raiva tem sido administrada ao longo da história política. Arjun Appadurai (2006) em seu livro 'Medo de Pequenos Números' destaca as emoções conflituosas entre as maiorias e as minorias oprimidas. Chantal Mouffe (2007) percebe o antagonismo entre as diferentes ideologias políticas como uma fonte ardente de política. A maior parte deste conhecimento emana das respostas emocionais ao 'onze de setembro' e a notável ameaça de terrorismo global (BUTLER, 2005; APPADURAI, 2006; SLOTERDIJK, 2008). De um modo semelhante a 'nova geopolítica do medo' surgiu dentro da sub disciplina de geopolítica crítica (MEGORAN, 2006; GREGORY; PRED 2007; SPARKE, 2007; PAIN; SMITH, 2008; PAIN, 2009), focando-se nas dimensões transnacionais de medo e como o medo é igualmente produzido e imaginado de um local no mundo a outro.

Neste artigo, eu argumento que vale a pena estudar as geografias emocionais do cotidiano da política local, a fim de compreender o papel constitutivo das emoções para a política. Como as campanhas eleitorais podem ser vistas como 'dramas de identidade e diferença' (SPENCER 2007), nas quais performances emocionais desempenham um papel crucial. Elas são um lugar ideal para estudar as geografias emocionais da política. Com base na pesquisa etnográfica realizada durante a campanha eleitoral de 2009 na política local do Equador, o artigo está interessado no papel que as emoções desempenham na constituição de espacialidades da política local nas sociedades pós-coloniais.

As campanhas recentes do Equador são caracterizadas pela presença crescente de mulheres, indígenas e afro equatorianas, que foram excluídos da cidadania política pós-colonialista, após a introdução de uma lei de cotas de gênero (VEGA UGALDE, 2005) e a implementação dos partidos indígenas na década de 1990 (LLUCO 2005; SÁNCHEZ-PARGA, 2007). O artigo está especialmente interessado nas geografias emocionais da campanha eleitoral produzidas pelos chamados 'novos' indivíduos políticos. Empregando o conceito de interseccionlidade (CRENSHAW 1989, 1994), a pesquisa empírica tem como objetivo compreender como as performances emocionais (coletivas) dentro das campanhas políticas resultam, abordam e desafiam as experiências pós-coloniais de racismo, patriarcalismo e classismo.

\section{Transpondo geografias emocionais e políticas}

Nos últimos anos tem havido um crescente interesse no papel da emoção e do afeto como foco para a constituição de espaço e lugar na geografia social e cultural (ANDERSON; SMITH, 2001; DAVIDSON; MILLIGAN, 2004; BONDI et. al., 2005; THIEN, 2005; PILE 2009; SMITH et al., 2009a;). Dada a importância das emoções na constituição do espaço político é surpreendente, no entanto, que sejam poucas as considerações com foco nas emoções na política a partir de uma perspectiva geográfica.

Construo meu argumento em dois grupos de literatura que abordam a relevância política das geografias emocionais: geografias emocionais dos movimentos sociais e abordagens feministas em relação às emoções. A primeira vertente se envolve com geografias de movimentos sociais que lidam com o papel das emoções como elementos chave no ativismo (BOSCO, 2004, 2006, 2007; OSLENDER, 2007; WRIGHT 2008). Este grupo de trabalho tem conexão com uma ampla gama de literatura em torno das emoções em movimentos sociais (SCOTT, 1990; YANG, 2000; GOODWIN, et al., 2001; KEMPER, 2001), que abordam sentimentos coletivos e a relação entre emoções, ativismo político e identidade, tais como grupos étnicos (WEST-NEWMAN, 2004;OSLENDER, 2007), movimentos feministas (TAYLOR; RUPP, 2002; HOIMES, 2004) e a maternidade (BOSCO, 2004). A pesquisa de Bosco $(2004,2006,2007)$ sobre o trabalho emocional das 'Madres de Plaza de Mayo', que protestaram contra a violação dos direitos humanos nas ditaduras da Argentina, lamentando pública e coletivamente a perda de seus filhos, é um exemplo interessante que mostra como o ativismo do movimento social gira em torno de identidades de gênero e, concomitantemente, com emoções generificadas.

Semelhante a Bosco, a maioria desses estudos se concentra na política de identidade, alegando que as emoções de identidade específicas, tais como: o amor maternal (TAYLOR; RUPP, 2002) ou a raiva póscolonial racializada (WEST-NEWMAN, 2004) estão no centro do movimento do ativismo social. 
Embora, como Pain (2009) destaca, os geógrafos políticos feministas até hoje tiveram pouco a dizer sobre o papel da emoção dentro da política. A geografia feminista em geral, tem se preocupado com a generificação das emoções, desde o seu início, pelo desafio dos polos binários público/privado, razão/emoção, etc. (BONDI, 1990; ROSE， 1993; BLUNT; ROSE, 1994). Embora inicialmente a generificação, tanto da emoção quanto da política, era a preocupação central da política feminista, considerações recentes de geografia (política) feminista enfatizam que seria etnocêntrico, se não racista, assumir que gênero é sempre e em qualquer lugar a base primária de opressão, perseguição, ou exclusão (MOHANTY, 1991; ANZALDÚA, 1987). Relações de classe, raça, casta, sexualidade, religião, nacionalidade, etnia e outros eixos de afiliação são potencialmente excludentes, discriminatórios, e até mesmo violentos (HYNDMAN 2004).

Estas experiências de exclusão, discriminação e violência generificadas, racializadas e sexualizadas, etc., resultam e provocam emoções, tanto em indivíduos como em grupos sociais. Conectando-se a um grupo mais amplo de trabalhos sobre as emoções dentro de estudos feministas e de gênero (NELSON, 1999; AHMED, 2004; HOLMES, 2004; NGAI, 2005). As geógrafas feministas trataram as emoções tão diferentes como os medos cotidianos (VALENTIM, 1989), medo do terrorismo global (RADCLIFFE, 2007; PAIN; SMITH, 2008;), a esperança por mudanças (SPARKE, 2007; WRIGHT, 2008), tristeza e saudade (PRATT, 2009), etc.. Geógrafas feministas, no entanto, não se envolvem apenas empiricamente com as emoções, mas contribuem com seu trabalho, crítica e debates em torno das geografias emocionais e afetivas (BONDI, 2005; BONDI et al., 2005; TOLIAKELLY, 2006 ).

De particular relevância para o presente estudo é a crítica de Tolia-Kelly (2006) ao pensamento universalista e a falta de autenticidade histórica na consideração de Nigel Thrift (2004), em particular, de uma 'política espacial de afeto' e as abordagens neuropsicológicas das geografias afetivas em geral. Ela argumenta que, devido às relações de poder históricas e espaciais, corpos racializados, generificados e sexualizados têm capacidades diferentes para experimentar e afetar o espaço social em torno deles. $\mathrm{O}$ argumento de Tolia-Kelly serve como ponto de partida conceitual para o meu esforço em conceituar as geografias emocionais sentidas, experimentadas, e exibidas em grupos racializados e generificados nos espaços políticos equatorianos. Baseando-se em observações etnográficas feitas enquanto acompanhava mulheres que concorriam como candidatas nas eleições locais, eu exploro a vantagem de uma perspectiva interseccional no estudo empírico das geografias emocionais.

\section{Pesquisando geografias emocionais a partir de uma perspectiva interseccional}

As feministas não brancas como Anzaldúa (1987), Crenshaw (1989), Mohanty (1991) desenvolveram o conceito de interseccionalidade para mostrar que as mulheres experimentam a opressão de forma diferente e em diferentes graus, dependendo como o gênero se cruza com raça, classe, orientação sexual e outras categorias de organização social. O conceito torna possível "pensar em raça, classe e gênero [e idade, localização, sexo, deficiência, etc... adição do autor] como diferentes estruturas sociais que cada pessoa experimenta simultaneamente" (VALENTINE, 2007, p. 13). Como a pesquisa interseccional concentra-se nas inter-relações entre diferentes sistemas de opressão, é particularmente adequado contemplar a concepção pós-colonial das emoções.

Há uma discussão intensa, tanto em estudos de gênero como em geografias feministas, em como pesquisar a interseccionalidade. Os estudos de caso (MCCALL, 2005) ainda são vistos como a forma mais viável de lidar com a complexidade da investigação interseccional. Geógrafas feministas frequentemente se concentram em um 'local' específico, como o local de trabalho (MCDOWELL, 2008) ou a comunidade (LOBO, 2010). A maioria das análises baseia-se em métodos de pesquisa qualitativa. Ao fazer isso, a interseccionalidade é pesquisada como 'experiência vivida' (VALENTINE, 2007; HOPKINS; NOBLE, 2009;). A narração de Valentine (2007) sobre Jeanette, uma mulher surda que experimenta sua surdez de maneira muito diferente ao longo de sua vida e em espaços diferentes, é um exemplo de como à identidades especiais é dada importância por indivíduos em determinados momentos e em contextos específicos. Enquanto essas narrações destacam a experiência individual de interseccionalidade, as interseccionalidades estruturais e discursivas são abordadas apenas de maneira implícita. Poucos estudos consideram que categorias como raça e gênero não são, essencialmente, uma questão da cor da pele e sexualidade, mas de exploração imperial e trabalho subjugado (MCCLINTOCK, 1995). Assim como a própria Valentine (2007, p. 19) destaca:

a teorização existente do conceito de interseccionalidade supervaloriza as habilidades dos indivíduos em produzir ativamente suas próprias vidas e subestima

\section{Carolin Schurr}


como a capacidade de promulgar algumas identidades e realidades em vez de outras, é altamente contingente nos espaços repletos de poder e através dos quais nossas experiências são vividas.

Proponho superar esse problema combinando abordagens estruturais, discursivas e performativas neste artigo. Baseado no trabalho de Winker e Degele (2011), eu desenvolvo uma abordagem de três níveis para analisar a interseccionalidade das emoções. Em primeiro lugar, destacando as desigualdades estruturais na sociedade equatoriana, eu contextualizo os sentimentos coletivos expressos pelos 'novos' indivíduos políticos com base nas injustiças e desigualdades. Em segundo lugar, eu analiso os discursos, normas e ideologias em nível de representação, a fim de mostrar como os discursos políticos em torno da racionalidade, masculinidade e brancura justificaram a exclusão de pessoas 'racializadas' e mulheres por considerá-los como muito emotivos. Em terceiro lugar, (re) construindo a importância da emocionalidade nas performances de identidade dos 'novos' indivíduos políticos, eu viso compreender como elas (re) produzem e desafiam as imaginações estereotipadas das emoções de identidades específicas através de suas performances.

Desigualdades estruturais, representações simbólicas e construções de identidade são expressadas através de práticas sociais (BOURDIEU, 1998). Metodologias etnográficas são uma forma possível para captar práticas. A metodologia aplicada reflete os recentes apelos para uma mudança de metodologias etnográficas na geografia política (feminista) (SHARP, 2004; MEGORAN, 2006; KOFMAN, 2008). Entre 2008 e 2010, passei um total de 12 meses nas três províncias de Esmeraldas, Chimborazo e Orellana, acompanhando mulheres políticas em suas atividades diárias e realizando entrevistas com elas. As entrevistas semiestruturadas e parcialmente biográficas com quarenta mulheres políticas locais, entre elas sete candidatas provinciais para a Assembleia Nacional, três (vice) chefes de província, três (vice) prefeitas, treze vereadoras, quatorze vereadoras rurais, bem como com quinze mulheres chefiando organizações locais de mulheres.

Além disso, acompanhei duas chefes de província, duas prefeitas e quatro vereadoras por várias semanas em suas atividades políticas diárias e, posteriormente, gravadas em vídeo as suas atividades de campanha, passei uma semana em cada província durante a campanha eleitoral em 2009. Uma vez que as mulheres notavam que eu tinha uma câmera, elas me pediam para gravar suas atividades. Fotografias e vídeos foram usadas mais tarde para (re) construir uma precisa descrição dos acontecimentos, focando no que os corpos das candidatas estavam fazendo, suas expressões faciais, suas interações e seus gestos, em vez de perguntar-lhes "como vocês se sentem?"

\section{Interseccionalidade estruturais como causa e alvo de sentimentos coletivos}

Cerca de 500 pessoas aceitaram o convite indígena e camponês do partido Pachakutik para as eleições primárias no Coliseu da Coca, uma capital provinciana amazonense. Apesar do calor, as pessoas estão esperando pacientemente até que de repente a música foi ligada e um músico local canta uma canção popular composta especialmente para Guadalupe Llori, a chefe da província de Orellana. Ela é uma das duas chefes; todas as outras 22 províncias são governadas por homens. Os olhos das pessoas ao meu redor brilham enquanto cantam a música da campanha com paixão, euforicamente agitando as bandeiras do arco-íris do partido indígena Pachakutik, batendo palmas sem parar, gritando "que vivam as mulheres". A canção é seguida por um grupo de dança das crianças indígenas, todo vestido em trajes tradicionais, dançando ao ritmo da cumbia. O primeiro a falar é um homem indígena. Ele dá boas-vindas a todos em kichwa. Como o meu kichwa é muito pobre eu só entendo algumas palavras, quando ele diz 'Alli punsha, Alli Shamushka... Indígenas kichwa, Shuar, waorani,.... Yupaichani. A mulher indígena perto de mim explica que ele está pedindo às pessoas para lutarem juntos contra as práticas discriminatórias do governo. Em seguida, um comitê eleitoral é nomeado e as pessoas são convidadas a inscrever-se na lista de candidatos. Três homens são voluntários, mas parece difícil encontrar o mesmo número de mulheres - uma exigência da lei eleitoral após a introdução da quota de gênero. Todos os candidatos são, então, convidados a apresentar um breve discurso. Marco Santi refere-se a seus antepassados e à libertação do domínio espanhol e conclui seu discurso com as palavras 'agora chegou o momento que nós, os Indígenas, podemos ter uma palavra a dizer no mundo 


\begin{abstract}
político". As pessoas gritam com entusiasmo, 'Que viva Marco Santi'. Maggali Orellana, uma mulher grávida usando brincos na cor do Pachakutik, acusa o atual chefe da provínciade discriminação contra os povos Amazonenses (tribos indígenas) só porque eles defendem a sua terra. Em seguida, o microfone é passado para uma mulher indígena. Kantapari Nuni parece muito nervosa e insegura. Ela faz o menor discurso, tropeçando nas palavras enquanto ela fala em espanhol, que não é sua língua materna. Em seguida, a votação acontece. (Notas de campo, Coca, Janeiro de 2009).
\end{abstract}

Observar a narração a partir de uma perspectiva interseccional, tanto a persistência de 'interseccionalidade estruturais' (CRENSHAW, 1994) como as transformações em curso que estão atualmente acontecendo na política equatoriana se tornam evidentes. Levando em conta que até 1979 os requisitos de alfabetização efetivamente excluía até 705 das mulheres (QUEZADA, 2009) e quase todos os indígenas de participar das eleições (Yashar, 2005), o evento (re) narrado que inclui tanto candidatos do sexo feminino como indígenas é um testemunho das transformações em curso nos espaços políticos póscoloniais em relação à extensão dos direitos de cidadania a grupos sociais que foram excluídos no (pós)-colonialismo. Os resultados da cota de gênero e o sucesso político do movimento indígena se tornam visíveis através de pessoas como Marco Santi, Maggali Orellana e Nuni Kantapari.

A interação entre a consolidação das organizações de mulheres, o apoio dado a elas por agências internacionais (não) governamentais, a década Internacional das Nações Unidas para mulheres e a ratificação Equatoriana da CEDAW (Convenção Internacional para a Eliminação de Todas as Formas de Discriminação contra a Mulher) em 1981, resultaram em uma longa luta por uma lei de cotas de gênero, finalmente aprovada em 2000 (VEGA UGALDE, 2005). Agora, os partidos políticos são obrigados a incluir $50 \%$ de mulheres nas listas de voto. Essa quota faz do Equador um pioneiro na implementação de políticas de gênero (BEDFORD, 2008). Embora os resultados eleitorais estejam longe de atingir a paridade, a participação política das mulheres tem aumentado significativamente em todos os níveis políticos. Mulheres mestiças e afro equatorianas, no entanto, têm se beneficiado de uma maior extensão da cota do que as mulheres indígenas que estão subrepresentadas, tanto em relação aos homens indígenas como nas mulheres não indígenas (RADCLIFFE et al, 2002).

Protestos em massa do movimento indígena durante a década de 1990 discutiram ardentemente a responsabilidade do Estado em corrigir as antigas injustiças racializadas. Como resposta às políticas neoliberais de exclusão social e à crise dos partidos políticos tradicionais, o movimento indígena formou um partido político eleitoralmente viável em 1996 (MACAS, 2002; LLUCO, 2005; LUCERO, 2008; MARTÍNEZ NOVO, 2009). O partido indígena Pachakutik tem feito muito sucesso nos municípios, onde se pretende construir uma política que contesta "a exclusão dos povos indígenas e de baixa renda da política formal" (RADCLIFFE et al., 2002, p. 290).

O surgimento e as ações de ambos os movimentos, que se cruzam em certos momentos e espaços, podem ser interpretados como processos de descolonização, como o aumento da presença de povos indígenas, afro equatorianos e mulheres na política representativa, desafiam a dominação (pós) colonial de indivíduos do sexo masculino e branco mestiço.

As lutas políticas de ambos os movimentos baseiam-se na emocionalizão de suas reivindicações políticas contra a discriminação estrutural e, concomitantemente, a emocionalização do espaço político. A narração exemplifica como espaços políticos são projetados de forma que invoca resposta emocional. O uso de elementos altamente simbólicos, como a língua indígena kichwa, roupas tradicionais e o arco-íris da bandeira wipala, que é um símbolo do Império Inca, junto com o aumento do uso da música tradicional e dança, reconstroem os espaços políticos, a fim de gerenciar e instrumentalizar sentimentos coletivos. A narração etnográfica evidencia como 'emoções coletivas' (KEMPER, 2001) resultam e tratam das interseccionalidades estruturais (pós) coloniais. A raiva contra a longa negligência das províncias Amazonenses refletem sentimentos (pós) coloniais sobre desigualdades econômicas racializadas, a raiva contra a exclusão dos povos indígenas do espaço político eleitoral remonta às ideias coloniais de cidadania, ou o medo das mulheres indígenas de falar em público, que resultam de desigualdades estruturais.

A interseccionalidade torna-se evidente no caso de mulheres indígenas como Kantapari Nuni, que não só tem que superar a ansiedade de falar em público, mas também o desafio de falar em uma língua que, muitas vezes, não é sua língua materna. As desigualdades de gênero e de raça em relação a anos de escolaridade e qualidade de educação, que diferem significativamente entre moças e rapazes e entre escolas bilíngues rurais (kichwa) e urbanas (mestiço) podem ser vistas como 
causa dessas ansiedades (GARCIA-ARACIL; WINTER, 2006). Uma falta de voz generificada e racializada foi intimamente ligada à injustiça social, desigualdade econômica e falta de poder político ao longo da história (pós) colonial do Equador. Como Howard (2010) destaca para a Bolívia, mesmo quando os políticos indígenas usam o espanhol, eles sofrem com a falta de acesso ao discurso dominante e uma notável falta de competência. Como a citação exemplifica, no entanto, os líderes indígenas se sentem cada vez mais confiantes e até mesmo orgulhos de usar kichwa em seus discursos públicos.

O uso do kichwa e as emoções que o acompanham são exemplos de como as emoções que foram trazidos à existência por meio de desigualdades estruturais podem ser convertidas em emoções de resistência e subversão. Assim, os espaços políticos que são trazidos à existência, por essas emoções, contestam os discursos hegemônicos que definem o político como branco mestiço, masculino e racional.

\section{Discursos em torno da brancura e da masculinidade dos espaços políticos do Equador}

Os imaginários da política no Equador foram dominados (pós) colonialidade, pela superioridade masculina e branca (RADCLIFFE; WESTWOOD, 1996), esclarecidas ideias da racionalidade masculina, e uma divisão público-privada, generificada e racializada (PATEMAN, 1988; MILLS, 1997). A emotividade das mulheres, indígenas e descendentes escravizados dos povos afrodescendentes, foi marcada como um perigo potencial para o julgamento político. Discursos da supremacia masculina e branca resultaram em uma lei eleitoral excludente ou um tributo indígena e eram, ao mesmo tempo, (re) produzidos através de práticas políticas cotidianas (PRIETO, 2004). Apesar da lei de cotas de gênero e a crescente presença de lideranças indígenas, os espaços políticos continuam a ser imaginados como discursivamente branco, mestiço e masculino e, concomitantemente, como espaços em que as mulheres e os povos indígenas estão literalmente "fora de lugar":

"É que... sempre política, toda a vida, a política sempre foi feita por homens - tudo seja as organizações das comunidades, seja nos bairros, seja nos partidos políticos, tudo é governado por homens. Disseramnos que não somos adequadas para sermos políticas pois somos muito histéricas, mal- humoradas, e afetivas." (Entrevista com vereadora, março de 2010).

As hierarquias (pós) coloniais entre emoção: pessoal e pensamento/razão: público relegam 'ser emocional' (AHMED, 2004) a determinados grupos generificados e racializados. Consequentemente, as lutas dos movimentos sociais das mulheres e povos no Equador têm sido frequentemente rejeitadas por serem muito emocionais e, portanto, não têm sido levadas a sério por governantes locais e nacionais.

Estes binários discursivos são ofuscados pelas performances emocionais do cotidiano dos homens no cenário político, por exemplo, durante os discursos populistas, onde insultam violentamente a oposição e gritam slogans políticos com entusiasmo para animar seus públicos (DE LA TORRE, 2000). Enquanto discursos dicotômicos em torno da racionalidade da masculinidade e da emotividade da feminilidade, constroem o cidadão masculino como sem emoção, é o indivíduo político masculino hegemônico que realmente define quais emoções são consideradas adequadas e quais são descartadas como sendo muito emocionais, muito femininas, na vida política diária. Um argumento semelhante pode ser feito por rebaixar os desempenhos políticos indígenas como primitivos, não suficientemente sofisticados e, portanto, de nunca serem capazes de atender às normas masculinas e branco mestiças, como uma representante das mulheres indígenas aponta em uma entrevista:

Eles nos dizem "por que os indios querem ser algo superior, se eles nem sequer sabem fazer um discurso político propriamente dito, nem mesmo os profissionais serão capazes de fazer qualquer coisa, eles vão apenas estar lá para enfeitar a imagem da candidato." (Entrevista da representante das mulheres indígenas, fevereiro de 2009).

A força dos discursos em torno da brancura e da masculinidade da política torna-se evidente quando se analisa estas citações de uma perspectiva interseccional e levando-se em conta que elas são narradas por mulheres que se auto identificam como indígenas. A primeira citação, destaca que a constituição de espaços políticos exclusivamente indígenas, como as organizações comunitárias também baseiam-se na ideia da superioridade masculina. A segunda citação, relata que os líderes indígenas ainda são considerados 'fora do lugar' por muitos mestiços. Assim, as mulheres indígenas são discriminadas 'primeiro por serem mulher e segundo, por causa de sua etnia' (Entrevista prefeita, fevereiro de 2008). 
Devido aos processos generificados e racializados sobre os 'Outros', as mulheres indígenas não se encaixam nas predominantes imaginações (pós) coloniais sobre uma pessoa política racionalizada e sem emoção que 'tem que ter olhos azuis, cabelo loiro e estatura europeia’ (ibid.).

Assim, as mulheres indígenas são 'atingidas' segundo as palavras de Crenshaw (1989) - pelos sistemas de bloqueio de racismo e de patriarcalismo, confrontando um "sistema político patriarcal e mono étnico", como afirma a primeira secretária indígena de Relações Exteriores, Nina Pacari (2005, p. 73).

Sua exclusão política é frequentemente justificada por argumentos generalizados que são racistas, generificados e agrupados, em torno da falta de educação e capacidades profisssionais das mulheres (indígenas) e, (portanto, falta de pensamento racional) ou pela ordem de gênero indígena complementar que concebe as mulheres como responsáveis pela reprodução da cultura indígena, incluindo o trabalho emocional dentro da família e da comunidade. As mulheres indígenas e mestiças da mesma forma, no entanto, têm implantado, estrategicamente, discursos maternalistas em torno da emotividade 'natural' das mulheres (HERRERA, no prelo). A crescente participação política das mulheres foi edificada por uma profunda crise política durante a década de 1990 , consistindo-se de desconfiança nas instituições políticas e vários golpes presidenciais (CAÑETE, 2000; RIVERA VÉLEZ; RAMÍREZ GALLEGOS, 2005).

As mulheres justificaram a sua participação política, por sua superioridade moral e emocional e, em consequência, sua capacidade de literalmente 'limpar' a 'sujeira' masculina, e espaços corruptos. Idealizando a maternidade, as mulheres são encorajadas a negar os seus próprios interesses (...). Esse sofrimento pelos outros é, muitas vezes, interpretado como sendo as mulheres mais capazes de 'sentir' as necessidades da comunidade. A maternidade também representa ideias de auto abnegação e a rejeição do interesse próprio. (CRASKE, 1999, p. 4).

Essa reformulação estratégica da emotividade das mulheres através do movimento de mulheres é um exemplo de como discursos (pós) coloniais em torno da racionalidade dos indivíduos políticos masculinos e mestiços são desafiados. Como resultado, as geografias emocionais dos espaços políticos são constantemente negociadas e exclusões (pós-) coloniais de certos grupos feminilizados e racializados dos espaços políticos tornam-se cada vez mais contestados.

\section{Performances emocionais, como parte de construções políticas identitárias}

A abordagem performativa para a interseccionalidade das geografias emocionais oferece a possibilidade de focar no realizar das emoções (NG; KIDDER, 2010). Ao demonstrar como os políticos destacam certas emoções generificadas, racializadas ou agrupadas, de acordo com o público ao qual eles buscam discursar e a reivindicação política que eles visam fazer, eu ilustro como eles desafiam a política de identidade essencialista ofuscando qualquer identidade fixa. Eu exemplifico meu argumento baseada em observações feitas enquanto acompanhava Maria Mosquera, Vice-Prefeita de Esmeraldas em sua campanha em um pobre bairro suburbano em Esmeraldas Costeiras.

Certa vez chegamos a um popular bairro, Maria, a vice-prefeita chega em outra pick up junto com seu filho. As pessoas começam com um coro eleitoral, dançando aos ritmos de "vota 15, vota Maria". O bairro com suas ruas de areia e pequenas casas de madeira parecia bastante silencioso e despovoado logo que chegamos, mas de repente torna-se vivo com as pessoas da equipe de campanha, que estão em todo lugar com suas camisas azuis e laranja e bonés de baseball. Na primeira casa, Maria é confrontada com um cartaz de propaganda do partido do governo, Alianza País, que faz campanha por um "novo socialismo". Quando duas mulheres abrem a porta após sua batida, Maria critica violentamente a falsidade do governo $e$ grita com insistência, enquanto seus gestos corporais e, especialmente, seu punho enfatiza sua raiva: 'Eu nasci com a organização política revolucionária, e você deveria saber que o movimento democrático popular é o único partido de esquerda verdadeiro, o único representante verdadeiro dos sectores populares. Nós, como mulheres pobres, mulheres trabalhadoras, mulheres camponesas, professoras, não podemos ser de qualquer outra classe, você não deveria votar para aqueles pelucones ${ }^{l}$ racistas do governo que nos chamam de "negros de merda". (Notas

\section{Carolin Schurr}




\section{de campo, Esmeraldas, em abril de 2009).}

Enquanto Maria, durante todo o dia e, em cada atuação, fala com uma voz muito alta e enérgica, na última parte de seu discurso, ela levanta sua voz ainda mais. Sua raiva e desgosto sobre o incidente de poucos dias atrás, quando o presidente Correa chamou $\mathrm{o}$ Prefeito afro equatoriano 'negro de merda', reativou sentimentos (pós) coloniais de discriminação, incorporados em um discurso pós-colonial da supremacia branca mestiça. À luz destes ataques verbais, Maria coloca-se como negra, um indicador que ela não recorre em outros momentos ${ }^{2}$. Em uma entrevista alguns meses depois, ela afirma: "Eu sou negra, mas eu não sou 'negrista' o que significa que ela se identifica como afro equatoriana, mas ela não é a favor de se fazer política de identidade sobre essa identidade racializada. Em vez disso, ela nega diferença racial em apoio à unidade de classe. A negação de Maria da significação étnica tem que ser entendida como parte da retórica socialista do seu partido, de um proletariado (sem raça) unificado. Sloterdijk (2008) mostra que a comunista "economia da raiva", criou um grupo proletário coletivo, por gerenciar sua raiva coletiva contra as elites econômicas. Ao posicionar-se como socialista, ela baseia-se nesses sentimentos coletivos gerenciados pelo comunista "Banco Mundial de raiva" (ibid., p. 170). Ao fazer isso, ela constrói a sua identidade política como uma identidade de classe em favor dos pobres. Como família, ela relata que pode ser considerada pobre, a sua referência emocional à sua infância pobre é uma tentativa de posicionar-se como representante da setores populares e evocar sentimentos comuns de raiva contra a institucionalizada injustiça econômica de classes específicas.

Maria, salienta ainda, em suas interações com as mulheres, as emoções da mulher e a maternidade, baseando-se em pressupostos de diferenças maternais feministas de que as mulheres têm determinados sentimentos, necessidades e interesses (SAPIRO, 1981) que só podem ser representados por mulheres:

\section{É importante que haja mulheres no município que nos respeitem, mulheres que sintam a dor do povo, a dor de sua irmã, mulheres que sintam as necessidades das mulheres (notas de campo, Esmeraldas, em abril de 2009).}

West (1999) mostrou que a experiência de violência, lesões e discriminação das mulheres e as emoções resultantes têm sido cruciais para a política feminista. Apesar de Maria não identificar-se como "feminista", ela baseia-se em estratégias feministas, "as quais se mobilizaram em torno da injustiça desta violência e a demanda política e ética para sua reparação" (AHMED, 2004, p. 172).

Mais adiante, referindo-se ao mesmo argumento de identidade política maternalista, ela salienta que as mulheres priorizam uma política de programas de trabalho que favorece os interesses das mulheres, como saúde e educação e, em geral, 'fazem' política de forma diferente devido à sua emotividade de gênero:

Nós, como mulheres pensamos de duas maneiras: com a lógica política, com a lógica profissional, mas também pensamos com a mente de uma mãe, com o coração de uma mulher, por exemplo, que não há apenas um parque, mas também flores, porque são as flores que decoram o parque. (Notas de campo, Esmeraldas, abril de 2009).

Como o exemplo de Maria ilustra, as emoções de vínculos se destinam a ser invocadas nos eleitorados exibindo uma 'consciência interseccional' (FREDERICK, 2010): Primeiro em relação à raça, por insinuar práticas racistas do partido do governo nacional e, portanto, os discursos em torno da superioridade branca mestiça. Segundo, no que diz respeito à classe, declarando-se como tendo crescido nas mesmas circunstâncias e, portanto, evocando sentimentos coletivos contra as desigualdades estruturais e, terceiro, em relação ao gênero, enfatizando a solidariedade da mulher. No decorrer do dia, ela (re) constrói a sua identidade de múltiplas maneiras. Cada construção da identidade situacional é acompanhada por um conjunto de emoções e sentimentos relacionados à si própria ou às experiências específicas vividas pelo eleitor. As emoções exibidas frequentemente associadas às suas diversas fontes como preconceitos racistas coloniais e pós-coloniais contra os negros, simultaneamente as experiências violentas e alegres de ser mulher, a retórica socialista de um proletariado unido e experiências de pobreza. Ao fazer isso, as performances emocionais esbarram nos rígidos limites de categorias de identidade fixa, estabelecendo relações temporárias e espacialmente únicas incorporadas entre Maria e as pessoas as quais ela fala. 


\section{Conclusão: pensando emoções a partir de uma perspectiva interseccional}

\author{
Sem paixões, sentimentos e afetos não \\ haveria geografia (política, acrescentado \\ pelo autor) digna deste nome. (SMITH et al, \\ 2009b, p. 10.).
}

Em um nível conceitual, o presente trabalho se propõe a ampliar as abordagens feministas nas geografias emocionais, integrando o conceito de interseccionlidade. Ao fazer isso, eu enfatizo a importância do apelo de Tolia-Kelly (2006) para uma geografia emocional "sensível às geometrias de poder". O estudo de caso empírico tem por objetivo traduzir sua afirmação teórica em prática de pesquisa, mostrando como as presentes relações de poder e suas emoções correspondentes dentro da sociedade equatoriana estão ligados à história da (pós) colonialidade, dominação, subordinação e resistência. Espaços políticos continuam a ser moldados por hierarquias sociais racializadas e generificadas. Em consequência, sua construção é influenciada pela forma nas quais corpos marcados experimentam e expressam emoções no espaço político. Uma perspectiva interseccional oferece a oportunidade de pesquisar os envolvimentos e as relações entre sentimentos e identidades coletivas. Ao mostrar o entrelaçamento (1) das desigualdades estruturais que levam à diferentes capacidades de experimentar e afetar o espaço político emocionalizado em torno deles, (2) discursos generificados, racializados e agrupados que constituem o espaço político, e (3) performances emocionais localizadas que tratam de emoções culturalmente significativas, eu apelo para a importância de levar em conta a reciprocidade entre estrutura, discurso e performatividade, ao se estudar as geografias emocionais.

Como os dados empíricos sugerem, uma análise interseccional de geografias emocionais precisa estar atenta à forma como categorias de identidade e relações de poder tornam-se relevantes em performances emocionais e como essas categorias se cruzam entre si. A consideração empírica desafia a proposta, muitas vezes criticada, estabilidade e o essencialismo da política de identidade (NOBLE, 2009). Ao perguntar porque exatamente essas intersecções tornam-se cruciais para as performances emocionais em específicas etapas políticas, uma melhor compreensão da relação entre identidade, emoção e política pode ser desenvolvida.
* Em primeiro lugar, agradeço a todas as mulheres que eu acompanhei durante suas campanhas eleitorais, as quais não se cansaram de me explicar as transcrições ocultas e públicas de suas ações políticas. Agradeço à comissão organizadora da Conferência da UGI sobre "Gênero e Geografia", no Rio de Janeiro, em 2011, por ter me convidado para apresentar este trabalho e traduzi-lo em Português. Agradeço a Martin Müller, Jonathan Everts, Katharina Abdo, e a dois peritos anônimos pelos comentários cuidadosos sobre o esboço deste artigo. Sou grata pelo financiamento do Swiss National Science Foundation (SNF) e pelo apoio recebido dentro da escola de graduação "gênero: os manuscritos e transcrições", realizado no Centro Interdisciplinar de Estudos de Gênero da Universidade de Berna. (Tradução de Silvana Hilgemberg)

1 O termo pelucones foi usado pelo presidente Correa para referir-se às forças de oposição da alta sociedade urbana em Guayaquil em um sentido pejorativo. Pelucón significa peruca e associa-se ao momento em que os membros da aristocracia nas colônias, como muitos outros aristocratas, usavam perucas. Ao chamá-los pelucón, Correa se refere às práticas egoístas e antiéticas das elites coloniais. É interessante que Maria usa esse termo para descrever o próprio Correa. Ela não está sozinha em sua crítica, já que ele vem de um grande família rica, além de ter sido educado nas melhores universidades e no exterior.

2 Mostrando como os candidatos, em certos momentos, colocam uma certa categoria de identidade em primeiro plano através de suas expressões emocionais e negligenciando-a completamente em outro momento.

\section{Referências}

AHMED, Sara. The cultural politics of emotion. Edinburgh: Edinburgh University Press, 2004.

ANDERSON, Kay; Smith, SUSAN, J. Editorial: emotional geographies. Transactions of the Institute of British Geographers, v. 26, n. 1, p. 7-10, 2001.

ANZALDÚA, Gloria. Boderlands: la frontera - the New Mestiza. San Francisco: aunt lute books, 1987.

APPADURAI, Arjun. Fear of small numbers: an essay on the geography of anger. Durham; London: Duke University Press, 2006.

BEDFORD, Kate. Governing Intimacy in the World Bank. In: RAI, Shirin M.; WAYLEN, Giorgia (Orgs.). 
Global governance: feminist perspectives. New York: Palgrave, 2008

BLUNT, Alison; ROSE, Gillian (Orgs.). Writing women and space: colonial and postcolonial geographies. New York: Guilford Press, 1994.

BONDI, Liz. Feminism, postmodernism, and geography: space for women?. Antipode, v. 22, n. 2, p. 156-167, 1990.

BONDI, Liz. Making connections and thinking through emotions: between geography and psychotherapy. Trans. Inst. Br. Geogr. N.S. n. 30, p. 433-448, 2005.

BONDI, Liz, et al. Introduction: geography's 'emotional turn'. In: DAVIDSON, Joyce, BONDI, Liz; SMITH, Mick (Orgs.). Emotional geographies. Aldershot: Ashgate, 2005.

BOSCO, Fernando J. Human rights politics and scaled performances of memory: conflicts among the "Madres de Plaza de Mayo" in Argentina. Social \& Cultural Geography, v. 5, n. 3, p. 381-402, 2004.

BOSCO, Fernando J. 'The Madres de Plaza de Mayo and three decades of human rights' activism: Embeddedness, emotions, and social movements. Annals of the Association of American Geographers, v. 96, n. 2, p. 342-365, 2006.

BOSCO, Fernando J. Emotions that build networks: geographies of human rights movements in Argentina and beyond. Tijdschrift voor Economische en Sociale Geografie, n. 98, p. 545-63, 2007.

BOURDIEU, Pierre. Practical reason: on the theory of action. Stanford: Stanford University Press, 1998.

BUTLER, Judith. Gefährdetes leben: politische essays. Frankfurt: Suhrkamp, 2005.

CAÑETE, María Fernanda. La crisis ecuatoriana: sus bloqueos económicos, políticos y sociales. Quito: CEDIME, 2000.

CRASKE, Nikki. Women and politics in Latin America. New Jersey: Rutgers University Press, 1999.

CRENSHAW, Kimberlé. Demarginalizing the intersection of race and sex: a black feminist critique of antidiscrimination doctrine. The University of Chicago Legal Forum, v. 1, n. 1, p. 139-167, 1989.
CRENSHAW, Kimberlé. Mapping the margins: intersectionality, identity politics, and violence against women of color. In: ALBERTSON FINEMAN, Martha; MYKITIUK, Rixanne (Orgs.). The public nature of private violence. New York: Routledge, 1994.

DAVIDSON, Joyce; MILLIGAN, Christine. Embodying emotion sensing space: introducing emotional geographies. Social \& Cultural Geography, v. 5, n. 4, p. 523-532, 2004.

DE LA TORRE, Carlos. Populist seduction in Latin America: the Ecuadorian experience. Ohio: Ohio University Press, 2000.

FREDERICK, Angela. Practicing electoral politics in the cracks: intersectional consciousness in a Latina candidate's city council campaign. Gender \& Society, n. 24, p. 475-498, 2010.

GARCIA-ARACIAL, Adela; WINTER, Carolyn gender and ethnicity differentials in school attainment and labor market earnings in Ecuador. World Development, v. 34, n. 2, p. 289-307, 2006.

GOODWIN, Jeff et al. (Orgs.). Passionate politics: emotions and Social Movements. Chicago, 2001.

GREGORY, Derek; PRED, Allan (Orgs.). Violent geographies: fear, terror and political violence. London: Routledge, 2007.

HERRERA, Gioconda. Sujetos y prácticas feministas en el Ecuador: 1980-2005. Quito: FLACSO, forthcoming, 2007.

HOHHET, Paul. Politics, identity, and emotion. Boulder: Paradigm Publishers, 2009.

HOLMES, Mary. Feeling beyond rules: politicizing the sociology of emotion and anger in feminist politics. European Journal of Social Theory, v. 7, n. 2, p. 209-228, 2004.

HOPKINS, Peter; NOBLE. Masculinities in place: situated identities, relations and intersectionality. Social \& Cultural Geography, v. 10, n. 8, p. 811-819, 2009.

HOWARD, Rosaleen. Language, signs, and the performance of power: the discursive struggle over decolonization in the Bolivia of Evo Morales". Latin American Perspectives, v. 37, n. 3, p. 176-194, 2010. 
HYNDMAN, Jennifer. Mind the gap: bridging feminist and political geography through geopolitics. Political Geography, v. 23, n. 3, p. 307-322, 2004.

KEMPER, Theodore D. A structural approach to social movement emotions. In: GODWIN, Jeff; JASPER, James M; POLLETTA, Francesca (Orgs.). Passionate politics: emotions and social movements. Chicago: University of Chicago Press, 2001.

KOFMAN, Eleonore. Feminist transformations of political geography. In: COX, Kevin, LOW, Murray; ROBINSON, Jennifer (Orgs.). The SAGE handbook of political geography. London: SAGE Publications, 2008.

LLUCO, Miguel. Acera del Movimiento de Unidad Plurinacional. In: ESCÁRZAGA, Fabiola; GUIÉRREZ, Raquel (Orgs.). Movimiento Indígena en Amércia Latina: resistencia y proyecto alternativa. Puebla, Mexico: Juan Pablos Editores, 2005

LOBO, Michele. Negotiating emotions, rethinking otherness in suburban Melbourne. Gender, Place \& Culture, v. 17, n. 1, p. 99-114, 2010.

LUCERO, José Antônio. Struggles of voices: the politics of indigenous representation in the Andes. Pittsburgh: University of Pittsburg, 2008.

MACAS, Luis. Lucha del movimiento indígena en el Ecuador. Boletín ICCI, n. 37, abr. 2002.

MARTÍNEZ NOVO, Carmen (Orgs.). Repensando los movimientos indígenas. Quito: FLACSO, 2009.

MCCALL, Leslie. The complexity of intersectionality. Journal of Women in Culture and Society, v. 30, n. 31, p. 1771-1800, 2005.

MCCLINTOCK, Anne. Imperial leather: race, gender and sexuality in the colonial contest. New York: Routledge, 1995.

MCDOWELL, Linda. Thinking through work: complex inequalities, constructions of difference and trans-national migrants. Progress in Human Geography, v. 32, n. 4, p. 491-507, 2008.

MEGORAN, Nick. The critical geopolitics of danger in Uzbekistan and Kyrgyzstan. Environment and Planning D - Society and Space, v. 23, n. 4, p. 555580,2005 .
MEGORAN, Nick. For ethnography in political geography: experiencing and re-imagining Ferghana Valley boundary closures. Political Geography, v. 25, n. 6, p. 622-640, 2006.

MILLS, Charles. The racial contract. Ithaca: Cornell University Press, 1997.

MOHANTY, Chandra Talpade. 'Under Western Eyes': Feminist scholarship and colonial discourse. In: MOHANTY, Chandra Talpade; RUSSO, Ann; Torres, Lourdes (Orgs.). Third World women and the politics of feminism. Bloomington: Indiana University Press, 1991.

MOUFFE, Chantal. Über das politische: wider die kosmopolitische Illusion. Frankfurt am Main: Suhrkamp, 2007.

NELSON, Diane M. A finger in the wound: body politics in Quincentennial Guatemala. Berkeley: University of California Press, 1999.

NG, Kwai; KIDDER, Jeffrey L. Toward a theory of emotive performance: with lessons from how politicians do anger. Sociological Theory, v. 28, n. 2, p. 193-214, 2010.

NGAI, Sianne. Ugly feelings. Cambridge: Harvard University Press, 2005

NOBLE, Greg. 'Countless acts of recognition': young men, ethnicity and the messiness of identities in everyday life. Social \& Cultural Geography, v. 10, n. 8, p. 875-891, 2009

OSLENDER, Ulrich. Revisting the hidden transcript: oral tradition and black cultural politics in the Colombian Pacific coast region. Environment and Planning D: Society and Space, v. 25, n. 6, p. 11031129, 2007.

PACARI, Nina. Case Study: ecuador - unfinished business. The political participation of indigenous women. In: IDEA (Orgs.). Women in Parliament: beyond numbers. Stockholm: IDEA, 2005.

PAIN, Rachel. Globalized fear? Towards an emotional geopolitics. Progress in Human Geography, v. 33, n. 4, p. 466-486, 2009.

PAIN, Rachel; SMITH, Susan. Fear: critical geopolitics and everyday life. Aldershot: Ashgate, 2008. 
PATEMAN, Carole. The sexual contract. Stanford: Stanford University Press, 1988.

PILE, Steve. Emotions and affect in recent human geography. Trans. Inst. Br. Geogr. N.S, v. 35, n. 1, p. 5-20, 2009.

PRATT, Geraldine. Circulating sadness: witnessing Filipina mothers stories of family separation. Gender, Place \& Culture: A Journal of Feminist Geography, v. 16, n. 1, p. 3-22, 2009.

PRIETO, Mercedes. Liberalismo y temor: imaginando los sujetos indígenas en el Ecuador postcolonial, 1895-1950. Quito: FLACSO, 2004.

QUEZADA, Alexandra. Del derecho al voto a la presencia de las mujeres en la vida política nacional. In: RODAS MORALES, Raquel (Orgs.). Historia del voto feminino en el Ecuador. Quito: CONAMU, 2009 .

RADCLIFFE, Sarah. Latin American indigenous geographies of fear: living in the shadow of racism, lack of development, and antiterror measures. Annals of the Association of American Geographers, v. 97, n. 2, p. 385-397, 2007.

RADCLIFFE, Sarah, et al. Reterritorialised space and ethnic political participation: indigenous municipalities in Ecuador. Space \& Polity, v. 6, n. 3, p. 289-305, 2002.

RADCLIFFE, Sarah; WESTWOOD, Sallie. Remaking the nation: place, identity and politics in Latin America. London: Routledge, 1996.

RIVERA VÉLEZ, Fredy; RAMÍREZ GALLEGOS, Franklin. Ecuador: democracy and economy in crisis. In: CRANDALL, Russell; PAZ, Guadalupe; ROETT, Riordan (Orgs.). The Andes in focus: security, democracy and economic reform. London: Lynne Rienner Publishers, 2005.

ROSE, Gillian. Feminism and geography: the limits of geographical knowledge. Oxford: Polity Press, 1993.

SÁNCHEZ-PARGA, José. El Movimiento Indígena Ecuatoriano: la larga ruta de la comunidad al partido. Quito: CAAP, 2007.

SAPIRO, Virgina. When are Interests Interesting? The Problem of Political Representation of Women. The
American Political Science Review, v. 75, n. 3, p. 701-716, 1981.

SCOTT, James. Domination and the arts of resistance: hidden transcript. Yale: Yale University Press, 1990.

SHARP, Joanne. Doing feminist political geographies. In: KOFMAN, Elemore; PEAKE, Linda; STAEHELI, Lynn (Orgs.). Mapping women, making politics: feminist perspectives on political geography. New York: Routledge, 2004.

SLOTERDIJK, Peter. Zorn und Zeit. Frankfurt: Suhrkamp, 2008.

SMITH, Adam. et al. (Orgs.). Emotion, place and culture. Farnham: Ashgate, 2009a.

SMITH, Mick, et al. Introduction: geography and emotion - emerging constellations. In: SMITH, Mick; DAVIDSON, Joyce; CAMERON, Deborah; BONDI, Liz (Orgs.). Emotion, place and culture. Farnham: Ashgate, 2009b.

SPARKE, Matthew. Geopolitical fears, geoeconomic hopes, and the responsibilities of geography. Annals of the Association of American Geographers, v. 97, n. 2, p. 338-349, 2007.

SPENCER, Jonathan. Anthropology, politics, and the state: democracy and violence in South Asia. Cambridge: Cambridge University Press, 2007.

TAYLOR, Verta; RUPP, Leila. Loving internationalism: the emotion culture of transnational women's organizations, 1888-1945. Mobilization, v. 7, n. 2, p. 141-158, 2002.

THIEN, Deborah. After or beyond feeling? A consideration of affect and emotion in geography. Area, v. 37, n. 4, p. 450-454, 2005.

THRIFT, Nigel. Intensities of feeling: towards a spatial politics of affect. Geografiska Annaler, v. 86, n. 1, p. 57-78, 2004.

TOLIA-KELLY, Divya. Affect - an ethnocentric encounter? Exploring the 'universalist' imperative of emotional/affectual geographies. Area, v. 38, n. 2, p. 213-217, 2006.

VALENTINE, Gill. The geography of women's fear. Area, v. 21, n. 4, p. 385-90, 1989. 
VALENTINE, Gill. Theorizing and researching intersectionality: a challenge for feminist geography. The Professional Geographer, v. 59, n. 1, p. 10-21, 2007.

VEGA UGALDE, Silvia. La cuota electoral en Ecuador: nadando a contracorriente en un horizonte esperanzador. In: LEÓN, MAGDALENA (Orgs.). Nadando contra la corriente: mujeres y cuotas políticas en los páises andinos. Quito: UNIFEM, UNFPA, FLACSO, 2005.

WEST-NEWMAN, Catherine Lane. Anger, ethnicity, and claiming rights. Multicultural Education Abstracts, v. 4, n. 1, p. 27-52, 2004.

WEST, Traci. C. Wounds of the spirit: black women, violence, and resistance ethics. New York: New York University Press, 1999.

WINKER, Gabrielle; DEGELE, Nina. Intersectionality as multi-level analysis: dealing with social inequality. European Journal of Women's Studies, v. 18, n. 1, p. 51-66, 2011.

WRIGHT, Sara. Practising hope: learning from social movement strategies in the Philippines. In: PAIN, Rachel; SMITH, Susan (Orgs.). Fear: critical geopolitics and everyday life. Aldershot: Ashgate, 2008 .

YANG, Guobin. Achieving emotions in collective action: emotional processes and movement mobilization in the 1989 chinese student movement. The Sociological Quarterly, v. 41, n. 4, p. 593-614, 2000.

YASHAR, Deborah. Contesting citizenship in Latin America: the rise of indigenous movements and the postliberal challenge. Cambridge: Cambridge University Press, 2005. 